\title{
Contemporary Approaches to Statistical Mechanical Probabilities: A Critical Commentary Part I: The Indifference Approach
}

\author{
Christopher J. G. Meacham \\ Forthcoming in Philosophy Compass
}

\begin{abstract}
This pair of articles provides a critical commentary on contemporary approaches to statistical mechanical probabilities. These articles focus on the two ways of understanding these probabilities that have received the most attention in the recent literature: the epistemic indifference approach, and the Lewis-style regularity approach. These articles describe these approaches, highlight the main points of contention, and make some attempts to advance the discussion. The first of these articles provides a brief sketch of statistical mechanics, and discusses the indifference approach to statistical mechanical probabilities.
\end{abstract}

\section{Introduction}

Statistical mechanics is generally taken to account for thermodynamic phenomena, such as the melting of ice cubes in warm water, the diffusion of milk in coffee, and so on. It does this by assigning very high probabilities to the phenomena we see (ice cubes melting in warm water, milk diffusing in coffee) and very low probabilities to phenomena we don't see (broken eggs coalescing into whole ones, objects spontaneously sliding across counters).

But how should we make sense of these probabilities? Most of the discussion in the recent literature has focused on two kinds of proposals. First, there are the indifference approaches, that take statistical mechanical probabilities to be measures of rational indifference. On these views, statistical mechanical probabilities are the credences that a rational agent would have regarding a system given only certain information about what the system is like. Second, there are the regularity approaches, that take statistical mechanical probabilities to be part of an optimally simple and informative description of what the world is like. On these views, statistical mechanical probabilities are sophisticated ways of encoding the frequencies of certain kinds of events.

The merits and demerits of these two kinds of views have been subject to a lot of analysis. In this pair of articles, I will sketch the outlines of this debate, highlight the main points of contention, and make a few attempts to advance the discussion. In this, the first of these articles, I'll provide a brief sketch of the statistical mechanics, and discuss 
the indifference approach to statistical mechanical probabilities. In the second article, I'll discuss the regularity approach to statistical mechanics, and describe some outstanding issues, and some areas where further research is needed.

Due to space restrictions, my discussion will be limited in several ways. First, I will restrict my attention to the indifference and regularity approaches just described, and thus will skip over some historically important approaches to statistical mechanical probabilities. A particularly noteworthy omission is the time-average interpretation of statistical mechanical probabilities developed by the Ehrenfest and Ehrenfest (1990), which is still endorsed by a number of physicists. Second, I will assume something like the Boltzmannian account of statistical mechanics proposed by Albert (2000). While this is the most widely discussed formulation of statistical mechanics in the recent literature, there are interesting alternatives. In both cases, I refer those with an interest in these issues to the more comprehensive discussions others have provided. ${ }^{1}$

\section{Statistical Mechanics: Background}

Let's restrict our attention to the simplest case: classical statistical mechanics and point particles. Here's a first take on how statistical mechanics assigns probabilities. First fix the static features of the world: the total energy, the number of particles, and so on. Second, construct a space of possibilities compatible with these static constraints, with each point of this space representing one of the possible configurations of positions and momenta that these particles could have. Call this the phase space picked out by the static constraints. We can call the points of this space microstates, and regions of this space macrostates. ${ }^{2}$ Given that the world is in some region $B$ of this space, the statistical mechanical probability of it being in some region $A$ is equal to the proportion of the $B$ region that $A$ occupies (where the sizes of these regions are determined by a particular measure called the "Liouville measure"). ${ }^{3}$

This characterization of statistical mechanics seems to get a lot right. ${ }^{4}$ If we look at a cup containing a half-melted ice cube in hot water, it will correctly predict that five minutes from now it's overwhelmingly likely that the ice cube will be completely melted, and the

\footnotetext{
${ }^{1}$ See Sklar (1993), Uffink (2007), Frigg (2008b), and the references provided therein.

${ }^{2}$ Three comments. First, the term "macrostate" is usually reserved for regions of phase space that have been picked out using certain thermodynamic parameters (temperature, pressure, etc). For convenience, I'm using the term more loosely here, to refer to any region of phase space.

Second, I'll employ the usual convention of using the terms "microstate" and "macrostate" to denote properties of worlds as well as regions of phase space. (Where the micro/macrostate-property $m$ is instantiated by a world iff the point in phase space corresponding to the world lies in the micro/macrostate-region $\mathrm{m}$.)

Third, although we've set things up by constructing the phase space of the world, one can also apply this apparatus to isolated subsystems of the world. (In these cases, we'll use a smaller phase space representing the degrees of freedom of the isolated system.) So I will sometimes speak in terms of "systems" instead of "worlds" in what follows.

${ }^{3}$ Some have argued that these precise assignments are implausible, and have advocated what amount to interval valued probabilities instead; see Goldstein (2001), Maudlin (2007) and Albert (2009) for discussions. Similarly, some have argued in favor of restricting the scope of statistical mechanics in some ways; see Leeds (2003) and Callender (2009). Both of these issues are largely orthogonal to our concerns, however, so I'll put them aside.

${ }^{4}$ Though some have raised worries regarding whether the theory does, in fact, yield the kinds of predictions and explanations described below. For a description of some of these worries, see Uffink (2007) and Frigg (2008b).
} 
water in the cup will be lukewarm. And it predicts this because, according to the Liouville measure, the vast majority of the microstates compatible with the macroscopic description just given will evolve into a no-ice-cube-and-lukewarm-water macrostate.

Likewise, this characterization of statistical mechanics seems to provide successful explanations for many of the entropy-increasing processes which we encounter: why milk diffuses when poured into coffee, why cups don't spontaneously slide from one part of a table to another, and so on. These explanations will be similar to the explanation just given. High entropy macrostates are much larger than low entropy macrostates, so an arbitrarily wandering microstate is much more likely to wander into a higher entropy macrostate than a lower entropy one. ${ }^{5}$

That said, this characterization of statistical mechanics also seems to get a lot wrong. Consider the question of what a cup containing a half-melted ice cube in hot water was like five minutes ago. According to the Liouville measure, the vast majority of the microstates compatible with this macrostate will have evolved from a no-ice-cube-and-lukewarmwater macrostate. So this characterization of statistical mechanics predicts that five minutes ago the cup was filled with lukewarm water. But this is the wrong answer: the correct answer is that five minutes ago there was an unmelted ice cube in a cup of very hot water.

The problem stems from the fact that the characterization we've provided is time symmetric. It predicts that systems had a higher entropy in the past in the same way that it predicts that systems will have a higher entropy in the future. But the world isn't time symmetric. Entropy increases toward the future, not toward the past. So it seems that this characterization of statistical mechanics will make all of the wrong predictions about what the past was like.

One might appeal to our evidence of what the past was like to avoid this conclusion: we remember putting the ice cube in warm water, for instance. But if we believe the statistical mechanical probabilities, none of the evidence we have access to-memories, photographs, books, fossils - should be believed. After all, statistical mechanics tells us that it's much more likely that our memories spontaneously formed from a skull full of brain soup than it is that they're reliable indicators of a lower entropy past, and that it's much more likely that our history books spontaneously formed from a higher entropy state than it is that they're accurate descriptions of a low entropy past. So, if statistical mechanics is to be believed, none of our evidence about the past should be trusted. ${ }^{6}$

If our credences match the statistical mechanical probabilities described above, then we'll make correct predictions about future thermodynamic behavior, but incorrect predictions about thermodynamic behavior in the past. If we want to believe that the world is like we think it is - that our evidence about the past is not vastly misleading, and so on-then we'll need to add something to our initial characterization of statistical mechanics.

The canonical response to this problem is to add the Past Hypothesis: a claim that the world was initially in some particular low entropy macrostate, the Past State. The Past State is a special low entropy macrostate which satisfies certain conditions, such as having microstates that will spread out over the accessible regions of phase space in a highly

\footnotetext{
${ }^{5}$ Though there are additional worries regarding whether statistical mechanics succeeds, or is even supposed to succeed, in explaining phenomena of this kind. See section 3.2 for discussion of a dissenting view with respect to the explanatory role of statistical mechanics, and see Frigg (2008b) for a discussion of some other kinds of worries.

${ }^{6}$ For a more detailed discussion of these issues, see Albert (2000) and North (2009b).
} 
scattered and evenly distributed way as it evolves over time. ${ }^{7}$ With the addition of the Past Hypothesis, everything seems to fall into place: we get the right predictions about both the past and the future, we can trust our memories, and so on. ${ }^{8}$

Note that in order for this to work, the Past Hypothesis needs to have a special status; despite its name, it can't be just a hypothesis about what the past is like. Here is why. The statistical mechanical probabilities place normative constraints on our credences; namely, our credences should line up with these probabilities in certain ways. ${ }^{9}$ Given the method of assigning probabilities described at the beginning of this section, the probability of the initial state being the Past State is tiny. It follows that we're rationally required to have a tiny credence in the initial state being the Past State as well. And we can't avoid this problem by saying that we're going to adopt the Past Hypothesis as a mere hypothesis, because the statistical mechanical probabilities make the adoption of this hypothesis rationally impermissible.

In order to get around this problem, we need to change what the statistical mechanical probabilities are. On the regularity approach, as we'll see, the statistical mechanical probabilities are given by the laws. In order to change these probabilities, we'll need to set things up so that the laws yield different values. The most straightforward way to do this is to add the Past Hypothesis to the laws and assign a probability of one to the initial state being the Past State.

On the indifference approach, on the other hand, the statistical mechanical probabilities are provided by an Indifference Principle. In order to change these probabilities, we'll need set things up so that this principle yields different values. The most straightforward way to do this is to take the Indifference Principle to provide a measure of rational indifference over the possibilities compatible with a given set of laws. We can then get this Indifference Principle to yield the desired probabilities by adding the Past Hypothesis to the laws.

In either case, the Past Hypothesis needs to be given a special status, such as lawhood, in order to do the work we want it to.

\section{The Indifference Approach}

The indifference accounts of statistical mechanical probabilities generally go as follows. ${ }^{10}$ First it is argued that some kind of Indifference Principle provides a constraint on rational credence. Then it is argued that this Indifference Principle yields values that line up with the canonical statistical mechanical probabilities. Finally, it's suggested that the statistical mechanical probabilities are nothing more than the values the Indifference Principle spits out. What we've been calling 'statistical mechanical probabilities' are just the credences that a rational agent ought to have when in a certain state of ignorance. In particular, the statistical mechanical probability of $A$ given $B$ is just the credence that a rational agent ought to have in $A$ if their total evidence consisted of all and only $B$, the static features of

\footnotetext{
${ }^{7}$ See Albert (2000) and Winsberg (2004a).

${ }^{8}$ Though a number of authors have raised worries regarding whether this appeal to the Past Hypothesis works as suggested; see Uffink (2002), Winsberg (2004a), Winsberg (2004b), Parker (2005) and Earman (2006).

${ }^{9} \mathrm{~A}$ more precise characterization of the normative constraints imposed by statistical mechanical probabilities is offered in Meacham (2005). Meacham (2005) assumes that these probabilities are chances, but the constraints will be the same if we adopt an indifference approach.

${ }^{10}$ Some recent defenders of this kind of approach include Schaffer (2007), Frigg (2008a) and Uffink (2009).
} 
the world, and the laws. ${ }^{11}$

Different versions of this approach have been subject to different criticisms. But the two most persistent objections concern (i) the justification for adopting this Indifference Principle and (ii) the ability of this approach to make sense of statistical mechanical explanations. Let's look at each of these worries in turn.

\subsection{Objection 1: The Indifference Approach's Normative Claims Are Unjustified}

The first standard objection to the indifference approach concerns how these principles, and their prescriptions, are justified. In particular, why should we think that the Indifference Principle in question has identified the correct constraints on rational belief?

There is a sizable literature detailing the problems with the various attempts to formulate and justify different versions of this Indifference Principle. In some cases the principles have been shown to be ambiguous, ill-formed or inconsistent, in others the justifications offered for these principles have been shown to rely on implausible assumptions or to be question begging. There are too many wrinkles in this debate to provide a concise summary; for details I refer the reader to the discussions provided by van Fraassen (1989), Howson and Urbach (2005), North (2009a), Uffink (2009) and the references therein. For our purposes, it suffices to say that no characterization or justification of these Indifference Principles has received wide-spread acceptance. ${ }^{12}$

Many have taken these difficulties to refute the indifference approach. Suppose, however, that the proponent of the indifference approach grants that no satisfactory justification for their Indifference Principle can be provided. And suppose that they take this Indifference Principle to be a primitive postulate. How bad would this be?

That would seem to depend on whether there are alternative approaches that can do better. And it's not clear that the alternative approaches are any better off in this respect. Consider, for example, the proponent of the regularity approach to statistical mechanical probabilities. She will take these probabilities to be lawfully governed chances that place a normative constraint on our credences via something like Lewis's (1986) Principal Principle. But providing a satisfactory justification of the Principal Principle has been a notoriously difficult thing to do, and no one has provided a justification for this principle that is widely accepted. Moreover, compelling arguments have been made for thinking that no satisfactory justification of this sort is possible. ${ }^{13}$

\footnotetext{
${ }^{11}$ For convenience, I'm taking both propositions and phase spaces to be set of worlds, so we can speak of macrostates and propositions interchangeably. In order to make this identification, I'm taking the 'static features' of the phase space to include all of the information independent of particle positions and momenta needed to specify the qualitative state of a world, so that each point in the phase space corresponds to a unique possible world (or a unique set of qualitatively identical worlds). This ensures that, no matter what the agent's qualitative evidence is, the macrostate/proposition $B$ can be fine-grained enough to encode it.

${ }^{12}$ Perhaps the most popular of these approaches is the one suggested by Jaynes (1983). But this approach faces some outstanding problems to which no widely-accepted solution has been offered; see Howson and Urbach (2005) for a critical assessment of this approach and some recent attempts to defend it.

${ }^{13}$ See Strevens (1999) for a broad ranging discussion of the kinds of problems facing attempts to justify this principle. See Hall (2004) for a discussion of some of the worries facing proponents of regularity approaches in trying to justify this principle. See Loewer (2004) and Hoefer (2007) for two attempts to circumvent these difficulties.
} 
If no further justification of the Principal Principle is possible, then the proponents of the regularity approach will need to adopt it as a primitive postulate. If so, then it appears that both the indifference approach and the regularity approach are on a par in this regard. Both endorse certain normative constraints on rational belief, and neither is able to provide a further justificatory story for why these normative constraints hold.

(Of course, we should be careful not to make too much of this point. Just because both approaches need to take these normative constraints as primitive postulates doesn't mean that both of these postulates are equally plausible. Consider: the hedonic utilitarian may take the injunction to maximize pleasure and minimize pain to be a primitive postulate. The anti-hedonic utilitarian may take the injunction to maximize pain and minimize pleasure to be a primitive postulate. But these two views are not equally plausible.)

The worries regarding how to formulate the Indifference Principle are pressing issues that the proponent of the indifference approach needs to resolve. ${ }^{14}$ In addition, there are real worries regarding the justifications that have been offered to support these principles. But although no convincing justification has yet been offered for the kind of Indifference Principle the indifference approach requires, it's not clear that this is a worry for the proponents of the indifference approach alone. Proponents of alternative views face similar justificatory worries. As a result, it's not clear that these justificatory difficulties should be taken as a mark against the indifference approach.

\subsection{Objection 2: The Indifference Approach Can't Recover Sta- tistical Mechanical Explanations}

The second standard objection to the indifference approach concerns its ability to recover statistical mechanical explanations. To get a feel for this worry, let's consider a typical statistical mechanical explanation. Suppose we have a cup of coffee, and we pour some milk into it. We know from experience that the milk will spread out until, in a minute or two, it is more or less evenly distributed throughout the coffee. Why does this happen?

A typical statistical mechanical explanation goes like this. Consider the macrostate compatible with what we know about the cup of coffee and the milk, at the moment after we've poured the milk in. An overwhelming majority of the microstates in this macrostate, according to the Liouville measure, will be ones which will evolve into a state in which the milk is more or less evenly distributed throughout the coffee. Thus when we pour milk into coffee, it's overwhelmingly likely that the milk will soon diffuse evenly throughout the coffee. This explains why we've always observed the milk in coffee diffusing: it's overwhelmingly likely to do so.

Note that statistical mechanical probabilities play a key role in this explanation. If the statistical mechanical probability of milk diffusing in coffee were very low instead of very high, statistical mechanics would not provide a satisfying explanation for why milk diffuses in coffee. ${ }^{15}$

But if we adopt the indifference approach to statistical mechanical probabilities, it's not clear that statistical mechanical probabilities can play this explanatory role. On the

\footnotetext{
${ }^{14}$ In this respect, the indifference approaches and the regularity approaches are not on a par. There has been a growing convergence of opinion regarding how to formulate the Principal Principle, and unproblematic versions of this principle have been formulated; for example, see Arntzenius (1995), Hall (2004), Meacham (2005) and Nelson (2009). The same is not true of the Indifference Principle.

${ }^{15}$ See Strevens (2000).
} 
indifference approach, statistical mechanical probabilities are a priori and necessary facts about what we ought to believe. And it doesn't seem that such facts could explain the occurrence of (particular or general) events-a posteriori and contingent facts about what is the actually the case. ${ }^{16}$

Here are two kinds of replies one might offer to this objection. First, one could maintain that the indifference approach can ground such explanations, and that the critics have simply failed to notice some of the ways in which empirical facts play a role in these explanations, even on the indifference approach. Second, one could reject the assumption that statistical mechanics provides explanations for thermodynamic phenomena, and thus maintain that there are no explanations for the indifference approach to recover. Let's look at each of these responses in turn.

The first reply to this explanatory objection maintains that the indifference approach can recover statistical mechanical explanations. According to this reply, those who have raised this objection have failed to notice some of the ways in which empirical facts- $a$ posteriori contingent facts about what is the actually case-do play a role in these explanations, even on the indifference approach. And when these facts are taken into consideration, the reply goes, it becomes clear that the indifference approach can ground these kinds of explanations.

What empirical facts have the critics failed to take into account? Let's consider three ways in which empirical facts might inform the statistical mechanical probabilities.

First, the Indifference Principle tells agents how to be indifferent in certain epistemic situations. But the epistemic situation of an agent is an empirical matter. So the statistical mechanical probability relevant to a given agent - the statistical mechanical probability with which she should align her credences-is determined in part by an empirical fact: what her evidence is. In particular, the probability relevant to an agent's credence in $A$ is the statistical mechanical probability of $A$ relative to $B$, where $B$ is the macroscopic description that encodes everything the agent knows about the world.

While this is true, it is of little help in responding to the original worry. The phenomenon to be explained - the explanandum-is the diffusion of milk in coffee. And since the macrostates $A$ and $B$ that pick out the relevant statistical mechanical probabilities are fixed by the explanandum, empirical facts concerning the evidence that agents have are irrelevant. ${ }^{17}$

Second, one might take empirical evidence to determine the measure we should be indifferent with respect to. But this claim is problematic. It's generally held that the beliefs of rational agents should satisfy conditionalization. And proponents of the indifference approach have generally endorsed this constraint. ${ }^{18}$ But if we accept conditionalization, then empirical evidence can't determine the measure we should be indifferent with respect to.

\footnotetext{
${ }^{16}$ Versions of this criticism have been raised by a number of people, including Sklar (1993), Albert (2000) and Loewer (2001).

${ }^{17}$ Of course the explanandum stated here is vague. But we can precisify the explanandum, and once we do, the relevant statistical mechanical probabilities will fall out, in a manner completely independent of agents or their evidence.

${ }^{18}$ One of the objections that has been raised against Jaynes' (1983) indifference approach is its purported incompatibility with conditionalization; see Friedman and Shimony (1971), van Fraassen (1981) and Uffink (1996). Jaynes' defenders have responded by arguing that these two positions are compatible, not by arguing that we should abandon conditionalization; see Williams (1980), Jaynes (1983) and Skyrms (1985).
} 
Given conditionalization, a rational agent's current credences are fixed by her initial credences and her current evidence. In this framework, credence constraints like those imposed by statistical mechanical probabilities can be translated into constraints on the initial credences of rational agents. So the indifference measure an agent is rationally required to use can change as the agent's evidence changes iff the initial credences an agent is rationally required to have can change as the agent's evidence changes. But the initial credences an agent is rationally required to have can't change as the agent's evidence changes. ${ }^{19}$ Thus the indifference measure an agent is rationally required to use can't depend on the agent's evidence.

Third, putting agents aside, it might be pointed out that the statistical mechanical probability relevant to an explanandum is picked out by empirical facts-the laws, the static features of the world, the relevant macrostates $A$ and $B$. And it might be suggested that these facts are what give the indifference approach's explanations the empirical bite they need. But this suggestion won't get us out of the problem. This is because we can set up explanations where all of these empirical facts are already provided by the explanandum. And this leads us back to our original worry-the worry of how $X$ (the explanans) can explain $Y$ (the explanandum) if $X$ expresses a priori and necessary facts about what we ought to believe, and $Y$ expresses empirical facts.

(To get a more concrete feel for why this suggestion won't work, consider the following example. Let $A$ be a macrostate in which milk has just been poured into a cup of coffee, and let $B$ be a macrostate containing all and only the microstates that will evolve into a macrostate in which milk is evenly diffused throughout the cup of coffee. Let $L$ express the dynamical laws of classical mechanics, let $S$ express the static features needed to pick out the phase space. Explanandum: "Given that the world was in an $L S A$-state, why was it in an $L S B$-state?" Explanans: "Because the probability of $L S B$ given $L S A$ is overwhelmingly high." On the indifference approach, the explanans is an a priori and necessary fact about what we ought to believe, while the explanandum is an a posteriori and contingent fact about what is actually the case.)

So it doesn't look like empirical evidence can inform statistical mechanical probabilities on the indifference approach in the right way to ground statistical mechanical explanations. Thus this first reply to the explanatory objection-that the indifference approach can recover statistical mechanical explanations-looks unpromising.

The second reply to the explanatory objection rejects the claim that statistical mechanics provides explanations for various thermodynamic phenomena, such as why ice cubes melt when placed in hot water. According to this reply, the only real explanation for why such events occur are the classical mechanical ones citing the initial conditions and the dynamical laws. And since there are no statistical mechanical explanations, the objection that the indifference approach fails to recover statistical mechanical explanations evaporates. ${ }^{20}$

It's worth appreciating how radical this claim is. Let's consider some of the implications of this reply.

First, note that this reply is at odds with the statistical mechanical explanations given in textbooks, and with the experiences most people have when they're offered statistical mechanical explanations. According to this reply, the only real explanation for why gasses diffuse is that the initial conditions of the universe were such that, given the dynamical

\footnotetext{
${ }^{19}$ This is because the diachronic constraints conditionalization imposes, and the agent's credences at different times, together ensure that the initial credences the agent is rationally required to have remains the same.

${ }^{20}$ See Schaffer (2007), Frigg (2008a), Uffink (2009) and Winsberg (2009).
} 
laws, gasses diffuse. Now consider the following textbook statistical mechanical explanation: the reason gasses diffuse is that the overwhelming majority of microstates in the relevant regions are ones which will soon evolve into states in which the gasses are diffused, and thus it's overwhelmingly likely that these gasses will soon end up in a diffused state. After being told this story for the first time, it seemed to me that I understood why gasses diffuse better than I did before. I already knew, of course, that gases consisted of particles, and that these particles interacted in certain ways, and that for these gases to end up diffusing the particles would need to have initially been in a state which would yield a diffused state given the dynamics. But it seemed to me that the statistical mechanical explanation provided me with a deeper understanding of why gasses diffuse. According to the suggested reply, this feeling is wholly illusory. When I heard the statistical mechanical explanation, I learned nothing new of explanatory value. And when textbooks offer such explanations, they do nothing to further one's understanding of the world.

Second, as Strevens (2000) notes, this reply makes it difficult to explain why statistical mechanics was accepted by the scientific community in the late 19th century. Statistical mechanics didn't make new predictions-people already knew that gases dispersed, and so on. And people were already aware of the microphysical explanations one might give for why a particular batch of gas molecules dispersed. So what did statistical mechanics contribute that led people to favor it over the rival theories being proposed at the time? The standard story is that statistical mechanics was able to provide a better explanation for thermodynamic phenomena than its competitors. And it explained them by assigning high probabilities to the thermodynamic phenomena we see, and low probabilities to the antithermodynamic phenomena we don't see. But if the suggested reply is correct, then the standard story can't be, since statistical mechanics doesn't provide anything of explanatory value. So this reply makes the acceptance of statistical mechanics by the scientific community something of a mystery.

Third, this stance undercuts more than just the usual statistical mechanical explanations. As Strevens (2000) notes, since many of the explanations of other disciplines depend on the usual statistical mechanical explanations, it seems these explanations will be undercut as well.

Here's an example. Recall the form of the statistical mechanical explanation for why ice cubes melt when placed in hot water-when ice cubes are put in hot water, there is a very high probability that such-and-such processes will occur, and these processes constitute the melting of ice cubes. According to the suggested reply, this is not a real explanation. The only real explanation for why ice cubes melt when placed in hot water is that the initial conditions were a certain way, and the dynamical laws then led to these phenomena occurring.

Now consider the standard explanation for why photosynthesis occurs when plants are exposed to sunlight. This explanation assumes that various biochemical reactions will occur, which in turn assumes that a number of thermodynamic processes will occur. And these are processes which, from the perspective of statistical mechanics, only have a very high probability of occurring. So the form of the standard explanation for why photosynthesis occurs when plants are exposed to sunlight is that when plants are exposed to sunlight, there is a very high probability that such-and-such processes will occur, and these processes constitute photosynthesis.

But if the standard statistical mechanical explanation for why ice cubes melt when placed in hot water isn't a real explanation because it relies on statistical mechanical prob- 
abilities, then the standard explanation for why photosynthesis occurs when plants are exposed to sunlight isn't a real explanation either. The only real explanation for why photosynthesis occurs when plants are exposed to sunlight is that the initial conditions were a certain way, and the dynamical laws then led to these phenomena occurring. And, of course, what goes for photosynthesis goes for most of the other explanations given in botany, as well as in zoology, physiology, neuroscience, food science, ecology, chemistry, and so on.

Likewise, if we follow this reply and discard statistical mechanical explanations, we're led to potential problems with quantum mechanical explanations. Given what we know, something like the Bohmian interpretation of quantum mechanics could be correct. And if something like this theory is true, then quantum mechanical probabilities will be like statistical mechanical ones: probabilities over initial conditions in a world governed by deterministic dynamics. So if statistical mechanical probabilities are of no explanatory value, then these quantum mechanical probabilities will be of no explanatory value either. And since many quantum mechanical explanations only explain phenomena by demonstrating why they are highly probable to occur, then we need to be prepared to discard many of our quantum mechanical explanations.

All told, this second reply to the explanatory objection threatens to lead to a kind of explanatory nihilism regarding most of the explanations the sciences have offered. This seems like a heavy price to pay.

There are a number of tricky issues regarding how to understand scientific explanation, especially probabilistic explanation. And very little of the work on scientific explanation has made a serious effort to assess the kinds of explanations that statistical mechanics seems to provide. ${ }^{21}$ As a result, it would be premature to draw any definitive conclusions regarding this objection and the two replies to it that we've considered. Nevertheless, the considerations examined here suggest that these explanatory worries pose a severe challenge to the tenability of the indifference approach. ${ }^{22}$

\section{References}

Albert, David. 2000. Time and Chance. Harvard University Press.

Albert, David. 2009. "Physics and Chance." Unpublished Manuscript.

Arntzenius, Frank. 1995. “Chance and the Principal Principle: Things Ain't What They Used To Be.” Unpublished Manuscript.

Callender, Craig. 2009. “The Past Histories of Molecules.” Unpublished Manuscript.

Earman, John. 2006. "The "Past Hypothesis": Not Even False." Studies in History and Philosophy of Modern Physics 37:399-430.

Ehrenfest, Paul and Tatiana Ehrenfest. 1990. The conceptual foundations of the statistical approach in mechanics. Dover.

\footnotetext{
${ }^{21}$ With Strevens (2009) being a recent exception.

${ }^{22}$ I would like to thank Phil Bricker, Maya Eddon, Barry Loewer and an anonymous referee for comments and discussion.
} 
Friedman, Kenneth and Abner Shimony. 1971. "Jaynes' maximum entropy prescription and probability theory." Journal of Statistical Physics 3:381-384.

Frigg, Roman. 2008a. "Chance in Boltzmannian Statistical Mechanics.” Philosophy of Science 75:670-681.

Frigg, Roman. 2008b. A Field Guide to Recent Work on the Foundations of Statistical Mechanics. In The Ashgate Companion to Contemporary Philosophy of Physics. Ashgate Press pp. 99-196.

Goldstein, Sheldon. 2001. Boltzmann's Approach to Statistical Mechanics. In Chance in Physics: Foundations and Perspectives. Springer-Verlag.

Hall, Ned. 2004. "Two Mistakes About Credence and Chance." Australasian Journal of Philosophy 82:93-111.

Hoefer, Carl. 2007. "The Third Way on Objective Probability: A Skeptic's Guide to Objective Chance.” Mind 116:549-596.

Howson, Colin and Peter Urbach. 2005. Scientific Reasoning: The Bayesian Approach. 3rd ed. Open Court Publishing Company.

Jaynes, Edwin. 1983. Papers on Probability, Statistics and Statistical Physics. Dordrecht: Reidel.

Leeds, Stephen. 2003. "Foundations of Statistical Mechanics-Two Approaches." Philosophy of Science 70:126-144.

Lewis, David. 1986. A Subjectivist's Guide to Objective Chance. In Philosophical Papers, Vol. 2. Oxford University Press.

Loewer, Barry. 2001. "Determinism and Chance." Studies in the History of Modern Physics 32:609-620.

Loewer, Barry. 2004. "David Lewis' Theory of Objective Chance." Philosophy of Science $71: 1115-1125$.

Maudlin, Tim. 2007. "What Could be Objective About Probabilities?" Studies in History and Philosophy of Modern Physics 38:275-291.

Meacham, Christopher J G. 2005. "Three Proposals Regarding a Theory of Chance." Philosophical Perspectives 19:281-307.

Nelson, Kevin. 2009. “On Background: Using Two-Argument Chance.” Synthese 1:165186.

North, Jill. 2009a. "An Empirical Approach to Symmetry and Probability." Forthcoming in Studies in History and Philosophy of Modern Physics.

North, Jill. 2009b. "Time and Thermodynamics." Forthcoming in The Oxford Handbook on Time. 
Parker, Daniel. 2005. "Thermodynamic Irreversibility: Does the Big Bang Explain What It Purports to Explain?" Philosophy of Science 72:751-763.

Schaffer, Jonathan. 2007. "Deterministic Chance?" The British Journal for the Philosophy of Science 58:113-140.

Sklar, Lawrence. 1993. Physics and Chance. Cambridge University Press.

Skyrms, Brian. 1985. "Maximum entropy inference as a special case of conditionalization." Synthese 63:55-74.

Strevens, Michael. 1999. "Objective Probability as a Guide to the World." Philosophical Studies 95:243-275.

Strevens, Michael. 2000. "Do Large Probabilities Explain Better?" Philosophy of Science 67:366-390.

Strevens, Michael. 2009. Depth: An Account of Scientific Explanation. Harvard University Press.

Uffink, Jos. 1996. "The constraint rule of the maximum entropy principle." Studies in History and Philosophy of Modern Physics 27:47-79.

Uffink, Jos. 2002. “Time and Chance." Studies in History and Philosophy of Modern Physics 33:555-563.

Uffink, Jos. 2007. Compendium to the Foundations of Classical Statistical Physics. In Handbook for the Philosophy of Physics. Elsevier pp. 924-1074.

Uffink, Jos. 2009. "Subjective Probability and Statistical Physics.” Forthcoming in Probabilities in Physics.

van Fraassen, Bas. 1981. "A problem for relative information minimizers in probability kinematics." British Journal for the Philosophy of Science 32:375-379.

van Fraassen, Bas. 1989. Laws and Symmetry. Oxford University Press.

Williams, P. M. 1980. "Bayesian conditionalization and the principle of minimum information." British Journal for the Philosophy of Science 31:131-144.

Winsberg, Eric. 2004a. "Can Conditioning on the "Past Hypothesis" Militate Against the Reversibility Objections?” Philosophy of Science 71:489-504.

Winsberg, Eric. 2004b. "Laws and Statistical Mechanics.” Philosophy of Science 71:707718.

Winsberg, Eric. 2009. "Laws and Chances in Statistical Mechanics." Forthcoming in Studies in History and Philosophy of Modern Physics. 